Article

\title{
Fatty Acids from Pool Lipids as Possible Precursors of the Male Marking Pheromone in Bumblebees
}

\author{
Edita Kofroňová, Adam Nekola, Josef Cvačka, Jiří Kindl and Irena Valterová * \\ Institute of Organic Chemistry and Biochemistry, Academy of Sciences of the Czech Republic, \\ Flemingovo nám. 2, Prague 166 10, Czech Republic; E-Mails: kofronova@uochb.cas.cz (E.K.); \\ adamnekola@centrum.cz (A.N.); cvacka@uochb.cas.cz (J.C.); kindl@uochb.cas.cz (J.K.) \\ * Author to whom correspondence should be addressed; E-Mail: irena@uochb.cas.cz; \\ Tel.: +420-220-183-298.
}

Received: 31 December 2013; in revised form: 14 February 2014 / Accepted: 14 February 2014 / Published: 21 February 2014

\begin{abstract}
Triacylglycerols (TGs) stored in the fat bodies of bumblebee males have a species-specific composition. The striking structural similarities between TG fatty acids (FAs) and components of the male marking pheromone in certain species led to the hypothesis that FAs may serve as precursors in pheromone biosynthesis. Here, we analysed TGs from B. ruderatus, B. bohemicus, and B. campestris. Nonadec-9-ene and icos-15-en-1-ol are the main components of $B$. ruderatus labial gland secretion, forming up to $92 \%$ of the gland extract. The corresponding icos-11-enic and icos-15-enic acids were found in TGs at levels higher than usual for bumblebee species. We found similar relationships in B. campestris and $B$. bohemicus. These results suggest that FAs might be precursors of aliphatic compounds in the male pheromones. Furthermore, we report for the first time the pheromone structure of B. ruderatus males.
\end{abstract}

Keywords: Bombus ruderatus; Bombus campestris; Bombus bohemicus; fat body; labial gland secretion; pheromone biosynthesis

Abbreviations: APCI-MS - atmospheric pressure chemical ionisation mass spectrometry; $\mathrm{CI}$-chemical ionisation; $\mathrm{CN}$-number of carbon atoms; $\mathrm{DB}$; number of double bonds; ECN-equivalent carbon number; FA — fatty acid; FAME - fatty acid methyl ester; FAR - fatty acyl-CoA reductase; FB — fat body; GC/MS — gas chromatography and mass spectrometry; HPLC — high performance liquid chromatography; LG—-labial gland; SD—standard deviation; TG—-triacylglycerol 


\section{Introduction}

Most insect species produce pheromones to attract mating partners. However, mating strategies differ widely among insect families. While in Lepidoptera (the best-studied insect family with regard to sex communication) sex pheromones are mostly released by females to attract males for mating, in bumblebees males attract young queens with a pheromone secreted by their labial gland (LG). Males use this pheromone for marking prominent objects on their flight routes (known as patrolling behaviour) [1-3]. These marked spots are then attractive for females, i.e., young queens. Components of the labial gland secretion have therefore been called male marking pheromone; however, the term sex pheromone is used by some authors for the same signal [4].

Bioassays performed by Bergman [5] proved that the LG extract of B. lapidarius males is behaviourally active and can attract conspecific females. Coppée and co-workers [6] have shown in B. terrestris that the attractivity of the LG extract differs with the male age in correlation with the LG secretion quantity and composition [7]. Thorough bioassay showing which components are responsible for the attractiveness of the LG secretion has however not been published. Earlier work showed that the main components of the male LG secretion elicit antennal responses in virgin queens [7] and are thus "hot candidates" for pheromone components. This previous study was performed with two species: B. terrestris and B. lucorum.

The chemical nature of bumblebee males' LG secretions has been studied extensively in species occurring in Europe [4,8-16]. Each species produces a specific blend of compounds [17,18]. The gland secretion primarily contains two types of compounds: straight-chain aliphatic alcohols, aldehydes, and esters as one type and isoprenoid compounds as the second type.

Little is known about pheromone biosynthesis in bumblebees as opposed to moths, where biosynthetic pathways are well-described and understood [19-21]. There are two potential metabolic pathways leading to aliphatic compounds: biosynthesis from common lipids in the body or de novo synthesis from acetate units [22]. The first paper discussing the biosynthesis of marking pheromone was based on analysis of compounds isolated from 22 bumblebee species [23]. The authors suggested that these compounds are produced from saturated FAs by the action of specific glandular desaturases. Considering the recent literature and our own experiments, it seems that both pathways are possible. Most experiments have been performed with three model species: B. terrestris, B. lucorum, and $B$. lapidarius. While B. lucorum and B. lapidarius produce only aliphatic compounds in their pheromone glands $[1,8,13]$, B. terrestris produces aliphatic and terpenic alcohols and esters $[7,8]$. De novo biosynthesis of pheromone components was demonstrated by in vitro experiments in two species, B. terrestris and B. lucorum [24]. Incubations of labial glands with ${ }^{14} \mathrm{C}$-labelled acetate led to formation of FAs, esters (both species), and terpenoids (B. terrestris). On the other hand, a large series of experiments with deuterium-labelled FAs of different chain lengths applied in vivo or administered with food gave rise to aliphatic alcohols (B. lapidarius) and fatty acid ethyl esters (B. lucorum) in the LGs, as well as labelled triacylglycerols (TGs) in the fat bodies (FBs) [25,26]. These results support the hypothesis that lipidic precursors are involved in pheromone biosynthesis.

TGs stored in the FBs of bumblebee males have species-specific compositions [27]. Both the amount and composition of TG undergo changes with male age and these changes correlate with the dynamics of the marking pheromone production (study in B. terrestris and B. lucorum) [28]. In some 
cases, striking structural similarity between the TG FAs and components of the male marking pheromone supports the hypothesis that FAs may serve as precursors in pheromone biosynthesis. Such similarities can be seen in B. pratorum [8,29], B. lapidarius [1,30], and B. confusus [11,30]. In B. pratorum, this similarity is particularly conspicuous; two major aliphatic pheromone components, octadec-11-en-1-ol ( $15 \%$ in the LG) and pentacosa-7,17-diene (7\% in the LG), may be formed in the LG from octadec-11enoic acid (15\% in the FB) and hexacosa-9,19-dienoic acid (5\% in the FB), respectively, by a simple structural modification [29]. The aim of this study is to search for other bumblebee species in which structural similarity between FAs and pheromone components supports the lipid precursor hypothesis for the biosynthesis of male marking pheromone. Specifically, we studied three species in detail: Bombus (Megabombus) ruderatus, B. (Metapsithyrus) campestris, and B. (Ashtonipsithyrus) bohemicus. Furthermore, chemical analysis of the LG secretion of $B$. ruderatus males and detailed analyses of TGs and FAs in the FBs of all three species are reported here for the first time.

\section{Results and Discussion}

In the LG extract of $B$. ruderatus males (14 specimens), we identified 36 compounds. The secretion consisted of two major components and a number of medium and minor ones (Figure 1). Components present in amounts exceeding 0.1\% (17 compounds) are shown in Table 1. Nonadec-9-ene dominated in the mixture (77\%), followed by icos-15-en-1-ol (15\%). Except for farnesol and its two esters, the secretion consisted of aliphatic compounds, mainly hydrocarbons.

Figure 1. Gas chromatogram of the labial gland extract of B. ruderatus males; 1-nonadec-9-ene; 2 - icos-15-en-1-ol.

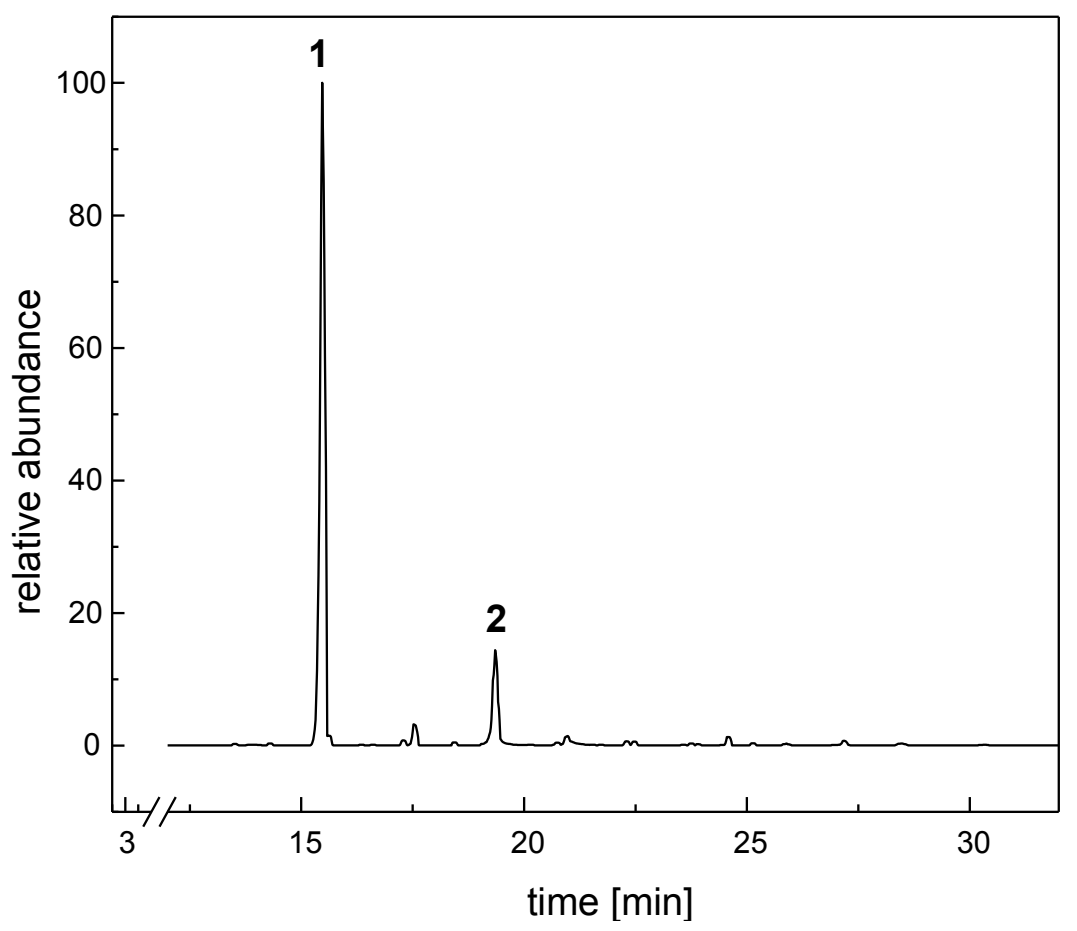

Saturated and unsaturated straight-chain hydrocarbons are always present in the LG extracts of bumblebee males. Their content usually ranges between $3 \%-10 \%$ with oxygenated compounds being the main secretion components. In B. ruderatus, however, nonadec-9-ene is the main secretion 
component (77\%), and it most probably plays a role in the chemical communication as has been shown earlier in other species for main LG components [7]. The presence of nonadec-9-ene as a component of male LG secretion seems to be typical for the whole subgenus Megabombus. While no species from the many other subgenera studied to date produce hydrocarbons as a main LG component, all Megabombus species have nonadec-9-ene as a major or medium component: B. hortorum, 20\% [8,31]; B. gerstackeri, 26\% [32]; B. consobrinus, 42\% [32]; B. portschinski, 46\% [33]; and B. argilaceus, $84 \%$ [33]. The occurrence of nonadec-9-ene seems to be a common trait in Megabombus, indicating that these species share a common biosynthetic pathway for pheromone formation.

Table 1. Composition (relative percent) of the labial gland secretion of B. ruderatus males $(\mathrm{N}=14)$. Components $<0.1 \%$ are not listed.

\begin{tabular}{|c|c|c|c|}
\hline Compound & Mean (\%) & Median (\%) & Standard deviation $(\%)$ \\
\hline$(E, E)$-Farnesol & 0.7 & 0.02 & 1.4 \\
\hline Octadec-9-ene & 0.2 & 0.1 & 0.2 \\
\hline Nonadec-9-ene & 76.7 & 78.0 & 8.4 \\
\hline Nonadecane & 0.9 & 0.6 & 0.5 \\
\hline Henicos-9-ene & 0.4 & 0.2 & 0.4 \\
\hline Henicosane & 1.2 & 0.8 & 0.8 \\
\hline Icos-15-en-1-ol & 15.0 & 16.4 & 8.3 \\
\hline Tricosane & 3.6 & 3.1 & 2.4 \\
\hline Pentacos-9-ene & 0.1 & 0.07 & 0.2 \\
\hline Pentacosane & 0.4 & 0.2 & 0.3 \\
\hline Docosen-1-ol ${ }^{\text {a }}$ & 0.1 & 0.0 & 0.1 \\
\hline Heptacos-9-ene & 0.2 & 0.1 & 0.4 \\
\hline Heptacosane & 0.1 & 0.1 & 0.1 \\
\hline Farnesyl tetradecenoate ${ }^{b}$ & 0.4 & 0.07 & 0.7 \\
\hline Hentriacont-9-ene & 0.1 & 0.1 & 0.1 \\
\hline $\begin{array}{c}\text { Farnesyl } \\
\text { octadecadienoate }\end{array}$ & 0.2 & 0.1 & 0.3 \\
\hline Icosenyl tetradecenoate $^{\mathrm{c}}$ & 0.2 & 0.05 & 0.5 \\
\hline
\end{tabular}

More than 60 different TGs have been identified in the TG fraction of FB lipid extract from B. ruderatus males (Table 2 and [27]). Similar to TGs from other bumblebee species, B. ruderatus TGs have 18:1 FA in highest abundance (TG 16:0_18:1_18:1 and TG 18:1_18:1_18:1). The retention order of TGs usually follows the equivalent carbon number, $\mathrm{ECN}(\mathrm{ECN}=\mathrm{CN}-2 \mathrm{DB}$, where $\mathrm{CN}$ is the number of carbon atoms and DB is the number of double bonds in a molecule). The ECN values for $B$. ruderatus TGs ranged between 40 and 60 (Table 2). In TGs with ECN $>50,20: 1 \mathrm{FA}$ was highly abundant (11\% of TGs contained the 20:1 moiety; Table 2, Figure 2). 
Table 2. Composition of triacylglycerols in the fat body of B. ruderatus males.

\begin{tabular}{|c|c|c|c|}
\hline ECN & CN:DB & Triacylglycerol (TG) $^{\mathrm{a}}$ & $\begin{array}{c}\text { Peak area relative \% } \\
(\text { mean } \pm \mathrm{SD} ; \mathrm{N}=4)\end{array}$ \\
\hline \multirow[t]{2}{*}{40} & $48: 4$ & 18:3_18:1_12:0; 18:3_14:0_16:1 & $0.1 \pm 0.1$ \\
\hline & $46: 3$ & 16:0_18:3_12:0; 14:0_14:0_18:3 & $0.1 \pm 0.1$ \\
\hline \multirow[t]{6}{*}{42} & $54: 6$ & $18: 1 \_18: 3 \_18: 2 ; 18: 2 \_18: 2 \_18: 2$ & $0.2 \pm 0.1$ \\
\hline & $52: 5$ & 18:3_18:1_16:1;18:2_18:2_16:1;18:2_16:0_18:3 & $0.8 \pm 0.2$ \\
\hline & $50: 4$ & 18:3_18:1_14:0; $18: 3 \_16: 0 \_16: 1$ & $0.8 \pm 0.2$ \\
\hline & $48: 3$ & 16:0_18:3_14:0;18:2_18:1_12:0; 18:2_14:0_16:1 & $0.4 \pm 0.4$ \\
\hline & $46: 2$ & 16:1_18:1_12:0; 16:1_16:1_14:0 & $0.1 \pm 0.1$ \\
\hline & $44: 1$ & 14:0_18:1_12:0; 14:0_16:1_14:0 & $0.1 \pm 0.1$ \\
\hline \multirow[t]{5}{*}{44} & $54: 5$ & 18:1_18:3_18:1 & $3.3 \pm 1.8$ \\
\hline & $52: 4$ & $18: 1 \_18: 3 \_16: 0$ & $5.1 \pm 2.1$ \\
\hline & $50: 3$ & 16:0_18:3_16:0; 18:2_16:1_16:0 & $1.6 \pm 0.2$ \\
\hline & $48: 2$ & 18:1_18:1_12:0;18:1_16:1_14:0; 16:1_16:1_16:0 & $1.3 \pm 0.5$ \\
\hline & $46: 1$ & 16:0_18:1_12:0;14:0_14:0_18:1 & $0.7 \pm 0.4$ \\
\hline \multirow[t]{5}{*}{46} & 56.5 & 18:1_18:1_20:3 & $0.7 \pm 0.3$ \\
\hline & $54: 4$ & 18:2_18:2_18:0; $18: 1 \_18: 1 \_18: 2$ & $2.2 \pm 0.2$ \\
\hline & $52: 3$ & 18:2_18:1_16:0;18:2_18:0_16:1;18:1_18:1_16:1 & $7.1 \pm 1.6$ \\
\hline & $50: 2$ & 18:1_18:1_14:0; 18:1_16:1_16:0 & $6.6 \pm 3.2$ \\
\hline & $48: 1$ & 16:0_18:1_14:0 & $2.4 \pm 1.4$ \\
\hline \multirow[t]{5}{*}{48} & $58: 5$ & $18: 2 \_18: 2 \_22: 1$ & $0.6 \pm 0.1$ \\
\hline & 56.4 & 18:1_18:1_20:2 & $0.5 \pm 0.2$ \\
\hline & $54: 3$ & $18: 1 \_18: 1 \_18: 1$ & $19.4 \pm 2.5$ \\
\hline & $52: 2$ & $18: 1 \_18: 1 \_16: 0$ & $22.8 \pm 4.7$ \\
\hline & $50: 1$ & 16:0_18:1_16:0 & $4.0 \pm 1.6$ \\
\hline \multirow[t]{3}{*}{50} & $56: 3$ & 18:1_18:1_20:1 & $4.9 \pm 2.5$ \\
\hline & $54: 2$ & $18: 1 \_18: 1 \_18: 0$ & $5.0 \pm 0.6$ \\
\hline & $52: 1$ & 18:0_18:1_16:0 & $1.4 \pm 0.5$ \\
\hline \multirow[t]{3}{*}{52} & $58: 3$ & 20:1_20:1_18:1;18:1_22:1_18:1 & $2.5 \pm 1.7$ \\
\hline & $56: 2$ & 20:1_18:0_18:1;18:1_18:1_20:0 & $1.1 \pm 0.7$ \\
\hline & $54: 1$ & 20:0_16:0_18:1;18:0_18:0_18:1 & $0.5 \pm 0.1$ \\
\hline \multirow[t]{3}{*}{54} & $60: 3$ & 20:1_20:1_20:1;20:1_22:1_18:1;18:1_18:1_24:1 & $0.9 \pm 0.8$ \\
\hline & $58: 2$ & 20:1_20:1_18:0; $18: 1 \_18: 1 \_22: 0$ & $0.9 \pm 0.7$ \\
\hline & $56: 1$ & 20:0_18:1_18:0 & $0.2 \pm 0.1$ \\
\hline \multirow[t]{3}{*}{56} & $62: 3$ & $18: 1 \_18: 1 \_26: 1$ & $0.5 \pm 0.5$ \\
\hline & $60: 2$ & $18: 1 \_18: 1 \_24: 0$ & $0.4 \pm 0.3$ \\
\hline & $58: 1$ & 22:0_18:1_18:0;18:0_26:1_14:0; 18:0_24:0_16:1 & $0.1 \pm 0.1$ \\
\hline \multirow[t]{2}{*}{58} & $64: 3$ & $18: 1 \_18: 1 \_28: 1$ & $0.2 \pm 0.2$ \\
\hline & $62: 2$ & 22:1_20:0_20:1; 18:1_18:1_26:0 & $0.2 \pm 0.2$ \\
\hline \multirow[t]{2}{*}{60} & $66: 3$ & 18:1_18:1_30:1 & $0.2 \pm 0.2$ \\
\hline & $64: 2$ & 18:1_18:1_28:0 & $0.2 \pm 0.1$ \\
\hline
\end{tabular}

a Shorthand notation of TGs according to Liebisch and co-workers [34]. The most likely FA in the $s n-2$ position of the glycerol is presented as second acid in the notation [28]. 
Figure 2. HPLC chromatogram of TG fraction of the fat body extract of B. ruderatus males. FA residues in TGs are abbreviated as follows (in alphabetic order): arachidic (A), gondoic $(\mathrm{G})$, linoleic $(\mathrm{L}), \alpha$-linolenic $(\mathrm{Ln})$, myristic $(\mathrm{M})$, oleic $(\mathrm{O})$, palmitic $(\mathrm{P})$, and stearic $(\mathrm{S})$.

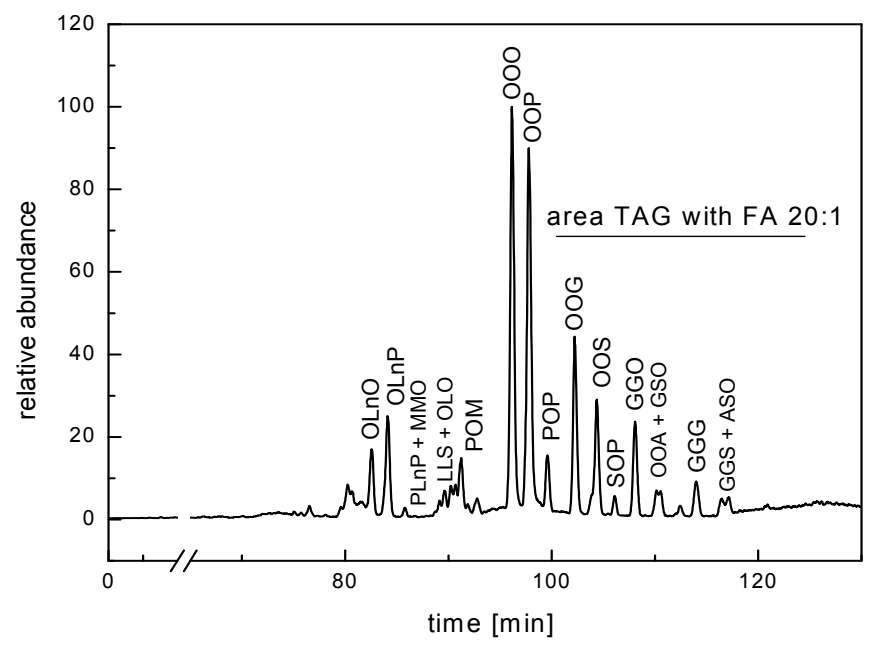

The highest content of 20:1 FA was in TGs bearing 18:1 or 18:0 acids, such as TG 18:1_18:1_20:1, TG 18:1_20:1_20:1, and TG 18:1_18:0_20:1. A TG with three 20:1 moieties, i.e., TG 20:1_20:1_20:1, was also detected. The relative amount of $20: 1$ acid methyl ester in the transesterified sample was $3.3 \%$ (Table 3). This amount is relatively high compared to those found in other, previously studied bumblebee species (usually below 1\% if present at all) [30].

Table 3. Relative proportions (in \%) of fatty acids in TG identified after a transesterification of TG fraction from B. ruderatus, B. campestris, and B. bohemicus fat body extracts.

\begin{tabular}{|c|c|c|c|c|}
\hline \multirow{2}{*}{ Fatty acid (Trivial name) } & \multirow{2}{*}{ Code } & \multicolumn{3}{|c|}{ Bombus species } \\
\hline & & ruderatus & campestris & bohemicus \\
\hline Decanoic (Capric) & $10: 0$ & 0.3 & trace & 0.1 \\
\hline Dodecanoic (Lauric) & $12: 0$ & - & 0.4 & 10.1 \\
\hline Tetradec-9-enoic (Myristoleic) & $9-14: 1$ & 0.2 & $0.1^{\mathrm{a}}$ & 6.0 \\
\hline Tetradecanoic (Myristic) & $14: 0$ & 1.3 & 1.6 & 15.6 \\
\hline Hexadec-9-enoic (Palmitoleic) & $9-16: 1$ & 5.1 & 1.2 & 14.3 \\
\hline Hexadec-11-enoic (Palmitvaccenic) & 11-16:1 & 1.1 & - & 2.3 \\
\hline Hexadecanoic (Palmitic) & $16: 0$ & 14.9 & 15.3 & 22.3 \\
\hline Octadecadienoic $^{\mathrm{a}}$ & $18: 2$ & 0.1 & 2.3 & 2.5 \\
\hline Octadec-9-enoic (Oleic) & $9-18: 1$ & 68.5 & 56.1 & 23.5 \\
\hline Octadec-11-enoic (cis-Vaccenic) & 11-18:1 & - & 4.3 & 1.2 \\
\hline Octadec-13-enoic (No trivial name) & 13-18:1 & trace & - & $0.1^{\mathrm{a}}$ \\
\hline Octadecanoic (Stearic) & 18:0 & 4.1 & 12.6 & 1.6 \\
\hline Icosadienoic $^{\mathrm{a}}$ & $20: 2$ & - & - & trace \\
\hline Icos-11-enoic (Gondoic) & 11-20:1 & 3.3 & 1.7 & $0.1^{\mathrm{a}}$ \\
\hline Icos-15-enoic (No trivial name) & 15-20:1 & trace & - & - \\
\hline Icosanoic (Arachidic) & 20:0 & 0.5 & 1.4 & 0.1 \\
\hline Docos-13-enoic (Erucic) & $13-22: 1$ & 0.5 & - & - \\
\hline Docosanoic (Behenic) & $22: 0$ & 0.1 & 0.4 & - \\
\hline Tetracosenoic $^{a}$ & $24: 1$ & 0.1 & - & - \\
\hline Tetracosanoic (Lignoceric) & $24: 0$ & trace & 0.2 & - \\
\hline
\end{tabular}

${ }^{a}$ Double bond position not determined (the diagnostic CI-MS fragments not found). 
To investigate a possible connection with pheromone biosynthesis, we determined the double bond position in 20:1 methyl ester using chemical ionisation mass spectrometry with acetonitrile as reaction gas [35]. The presence of diagnostic fragments $\mathrm{m} / \mathrm{z} 208$ and $\mathrm{m} / \mathrm{z} 280$ indicated the presence of a double bond in position 11 (gondoic acid methyl ester). Formation of nonadec-9-ene from gondoic acid via decarboxylation is a potential pathway to pheromone biosynthesis (Scheme S1) [19].

Another isomer of 20:1 methyl ester was present in the transesterified sample in a much smaller proportion. The CI spectrum showed fragments $\mathrm{m} / \mathrm{z} 152$ and $\mathrm{m} / \mathrm{z} 336$, corresponding to a double bond at position 15 in the 20:1 methyl ester. The chromatographic separation of the $\Delta 11$ and $\Delta 15$ isomers was, however, poor. The small peak corresponding to the $\Delta 15$ isomer was nearly hidden under the peak of the prevailing $\Delta 11$ isomer. Thus, the diagnostic fragments in CI-MS of the $\Delta 15$ isomer were not as pronounced as those in the spectrum of the $\Delta 11$ isomer. Formation of alcohols from fatty acids by the action of specific glandular fatty-acyl reductases has been proposed [36]. Icos-15-en-1-ol is present in the LG secretion in a lesser amount than nonadec-9-ene (1:5 ratio), which correlates with the excess of the $\Delta 11$ isomer of 20:1 acid that we observed in TGs.

Since nonadec-9-ene occurs in all Megabombus species studied so far, it would be interesting to investigate the lipid composition in more species of this subgenus to see whether the similarity between lipids and pheromone components found in B. ruderatus is a general pattern in Megabombus. To date, B. hortorum is the only other Megabombus species for which an analysis of TGs has been reported. The proportion of gondoic acid in the TG fraction of B. hortorum was rather low (0.7\%). B. hortorum, however, produces a rather complex mixture in the LG secretion [8], in which both aliphatic and terpenic compounds are present. Thus, the enzymatic biosynthetic system in $B$. hortorum is also likely rather complex.

Encouraged by the results with B. ruderatus, we focused on other bumblebee and cuckoo-bumblebee species that produce aliphatic compounds as their major LG secretion components. Of the species from which we had previously analysed LG secretions, we selected two as candidates for a detailed study of TG composition and FA structure: B. campestris and B. bohemicus. The LG secretion of B. campestris males contains icos-11-en-1-ol (21\%) and its corresponding acetate (1.3\%), icos-11-enal (9\%), octadec-11-enal (25\%), and octadec-11-en-1-ol (7\%) and its acetate (1\%) [14]. If the lipid precursor hypothesis holds true (Scheme S1), we would expect to see high levels of icos-11-enoic (gondoic) acid and octadec-11-enoic (cis-vaccenic) acid in TGs (Table 4). In fact, the relative amounts were 4.3\% cis-vaccenic acid and 1.7\% gondoic acid (Table 3). Considering that the main role of TGs in the FBs is to provide a pool of energy mostly for the flight muscles, the amount of specific FAs available for the production of secondary metabolites such as pheromones is quite substantial. Furthermore, Tomčala and co-workers [37] showed in Locusta migratoria that FB FAs with chains longer than 18 carbons are not mobilised in response to adipokinetic hormone application. Thus, longer chain FAs might play roles other than participation in primary metabolism and energy supply in bumblebees as well.

The LG secretion of $B$. bohemicus males is dominated by hexadec-11-en-1-ol (65\%) and the corresponding hexadec-11-enal (5\%) [14,38]. Octadec-11-en-1-ol and octadec-13-en-1-ol, together with the corresponding aldehydes, form minor secretion components (altogether $3 \%$ ). The $\mathrm{C}_{20}$ chain is of medium abundance and is mostly represented by icosa-11,15-dienal (14\%) with minor amounts of monounsaturated $\mathrm{C}_{20}$ derivatives such as icosa-11-en-1-ol, icosa-15-en-1-ol, and the corresponding icosenals, forming altogether $2 \%$ of the secretion mixture. Most of the expected FAs were found in FB 
TGs (Table 3 and Table 5) in various quantities. Palmitvaccenic (11-16:1) and cis-vaccenic (11-18:1) acids together formed 4\% of the FA mixture. Almost 23\% of TGs bore 20:1 and/or 20:2 FAs (Table 5).

Table 4. Composition of triacylglycerols in the fat body of B. campestris males.

\begin{tabular}{|c|c|c|c|}
\hline $\mathbf{E C N}$ & CN:DB & Triacylglycerol (TG) ${ }^{a}$ & $\begin{array}{c}\text { Peak area relative \% } \\
(\text { mean } \pm \mathrm{SD} ; \mathrm{N}=5)\end{array}$ \\
\hline \multirow{3}{*}{40} & $54: 7$ & $18: 2 \_18: 3 \_18: 2 ; 18: 3 \_18: 3 \_18: 1$ & $0.1 \pm 0.1$ \\
\hline & $52: 6$ & $18: 2 \_18: 3 \_16: 1 ; 18: 3 \_18: 3 \_16: 0$ & $0.1 \pm 0.1$ \\
\hline & $48: 4$ & 12:0_18:2_18:2 & $0.1 \pm 0.0$ \\
\hline \multirow{4}{*}{42} & $54: 6$ & $18: 2 \_18: 2 \_18: 2 ; 18: 3 \_18: 1 \_18: 2$ & $0.2 \pm 0.2$ \\
\hline & $52: 5$ & $18: 2 \_18: 2 \_16: 1 ; 16: 1 \_18: 3 \_18: 1$ & $0.3 \pm 0.2$ \\
\hline & $50: 4$ & 14:0_18:2_18:2; 18:1_14:0_18:3 & $0.3 \pm 0.1$ \\
\hline & $48: 3$ & $16: 1 \_16: 1 \_16: 1 ; 16: 0 \_18: 3 \_14: 0 ; 18: 2 \_14: 0 \_16: 1$ & $0.1 \pm 0.0$ \\
\hline \multirow{5}{*}{44} & $54: 5$ & $18: 1 \_18: 3 \_18: 1$ & $7.4 \pm 4.0$ \\
\hline & $52: 4$ & $18: 1 \_18: 3 \_16: 0$ & $6.1 \pm 2.4$ \\
\hline & $50: 3$ & $16: 1 \_14: 0 \_20: 2 ; 16: 1 \_16: 1 \_18: 1 ; 16: 0 \_16: 1 \_18: 2$ & $0.2 \pm 0.1$ \\
\hline & $48: 2$ & $18: 1 \_18: 1 \_12: 0$ & $0.4 \pm 0.1$ \\
\hline & $46: 1$ & 12:0_18:1_16:0;14:0_14:0_18:1;16:0_16:1_14:0 & $0.3 \pm 0.1$ \\
\hline \multirow{5}{*}{46} & $56: 5$ & 18:2_18:2_20:1; 18:1_18:1_20:3; 18:1_18:2_20:2 & $0.7 \pm 0.2$ \\
\hline & $54: 4$ & 18:1_18:1_18:2;18:3_18:1_18:0 & $3.8 \pm 0.7$ \\
\hline & $52: 3$ & $16: 1 \_18: 1 \_18: 1 ; 16: 0 \_18: 1 \_18: 2$ & $2.7 \pm 0.2$ \\
\hline & $50: 2$ & 18:1_14:0_18:1; 16:0_18:1_16:1 & $3.4 \pm 0.4$ \\
\hline & $48: 1$ & $16: 0 \_16: 1 \_16: 0 ; 14: 0 \_18: 1 \_16: 0$ & $0.6 \pm 0.2$ \\
\hline \multirow{3}{*}{48} & $54: 3$ & $18: 1 \_18: 1 \_18: 1$ & $24.5 \pm 2.3$ \\
\hline & $52: 2$ & 16:0_18:1_18:1 & $27.4 \pm 2.3$ \\
\hline & $50: 1$ & 16:0_18:1_16:0; 14:0_18:0_18:1 & $1.3 \pm 0.5$ \\
\hline \multirow{3}{*}{50} & $56: 3$ & 18:1_20:1_18:1 & $2.1 \pm 1.0$ \\
\hline & $54: 2$ & 18:1_18:0_18:1; 18:0_18:2_18:0 & $11.6 \pm 2.3$ \\
\hline & $52: 1$ & $16: 0 \_18: 1 \_18: 0 ; 18: 0 \_16: 1 \_18: 0$ & $2.7 \pm 1.2$ \\
\hline \multirow{3}{*}{52} & $58: 3$ & 18:1_20:1_20:1 & $0.1 \pm 0.1$ \\
\hline & $56: 2$ & 18:1_20:0_18:1 & $1.6 \pm 0.7$ \\
\hline & $54: 1$ & 18:0_18:0_18:1 & $1.1 \pm 0.5$ \\
\hline
\end{tabular}

${ }^{\text {a }}$ Shorthand notation of TGs according to Liebisch and co-workers [34]. The most likely FA in the $s n-2$ position of the glycerol is presented as second acid in the notation [28].

Enzymes catalysing reduction of fatty acids (FARs) in bumblebees would certainly deserve investigation in the future. So far, not many studies are devoted to insect reductases [36]. Most of known FARs catalyse FAs reduction to alcohols, aldehydes being intermediates of this reaction. In bumblebee labial glands however, aldehydes occur in high amounts in some species. Thus, some of aldehyde-generating FARs can be expected to operate in the male labial glands. Such enzymes were so far isolated only from plants or algae [39].

Earlier studies of TGs in lipids of male bumblebees were focused on the distribution of different TGs with regard to species-specificity. Intact TGs have been analysed and reported [27] without transesterification and determination of double bond positions. Here, we report these details for three 
species (one bumblebee and two cuckoo-bumblebee species), showing that species-specific differences also occur on the level of FA structures.

Table 5. Composition of triacylglycerols in the fat body of B. bohemicus males.

\begin{tabular}{|c|c|c|c|}
\hline ECN & CN:DB & Triacylglycerol (TG) ${ }^{a}$ & $\begin{array}{c}\text { Peak area relative \% } \\
(\text { mean } \pm \mathrm{SD} ; \mathbf{N}=6)\end{array}$ \\
\hline \multirow{6}{*}{38} & $54: 8$ & $18: 3 \_18: 3 \_18: 2$ & $0.1 \pm 0.0$ \\
\hline & $52: 7$ & $16: 1 \_18: 3 \_18: 3$ & $0.2 \pm 0.0$ \\
\hline & $50: 6$ & $14: 0 \_18: 3 \_18: 3$ & $0.2 \pm 0.0$ \\
\hline & $48: 5$ & $14: 1 \_16.1 \_18: 3$ & $0.1 \pm 0.0$ \\
\hline & $46: 4$ & 12:0_16:1_18:3 & $0.2 \pm 0.1$ \\
\hline & $44: 3$ & $14: 0 \_12: 0 \_18: 3$ & $0.2 \pm 0.1$ \\
\hline \multirow{6}{*}{40} & $54: 7$ & 18.3_18:1_18:3; 18:2_18:3_18:2 & $1.0 \pm 0.3$ \\
\hline & $52: 6$ & 18:3_18:3_16:0 & $1.0 \pm 0.3$ \\
\hline & $50: 5$ & $16: 1 \_16: 1 \_18: 3$ & $0.8 \pm 0.2$ \\
\hline & 48:4 & 12:0_18:3_18:1; & $1.9 \pm 0.7$ \\
\hline & $46: 3$ & 16:1_16:1_14:1; 16:0_18:3_12:0 & $0.9 \pm 0.3$ \\
\hline & $44: 2$ & $16: 1 \_16: 1 \_12: 0$ & $0.2 \pm 0.1$ \\
\hline \multirow{6}{*}{42} & $54: 6$ & $18: 2 \_18: 2 \_18: 2 ; 18: 3 \_18: 1 \_18: 2$ & $1.3 \pm 0.3$ \\
\hline & $52: 5$ & 18:2_18:2_16:1;16:1_18:3_18:1 & $4.5 \pm 0.5$ \\
\hline & $50: 4$ & 14:0_18:2_18:2; 16:1_16:1_18:2; 18:3_16:0_16:1 & $5.6 \pm 1.0$ \\
\hline & $48: 3$ & 16:1_16:1_16:1;14:0_14:1_20:2; 14:0_16:0_18:3 & $2.5 \pm 0.9$ \\
\hline & $46: 2$ & 16:1_18:1_12:0; 16:1_16:1_14:0 & $1.1 \pm 0.6$ \\
\hline & $44: 1$ & 12:0_18:1_14:0; $14: 0 \_16: 1 \_14: 0 ; 12: 0 \_16: 1 \_16: 0$ & $0.9 \pm 0.4$ \\
\hline \multirow{5}{*}{44} & $54: 5$ & 18:2_18:1_18:2; 18:1_18:3_18:1 & $9.4 \pm 4.2$ \\
\hline & $52: 4$ & 18:3_18:1_16:0;18:2_18:2_16:0; 16:1_16:1_20:2 & $12.0 \pm 3.2$ \\
\hline & $50: 3$ & 16:1_16:1_18:1;14:0_16:0_20:2; 16:0_16:0_18:3 & $4.6 \pm 1.4$ \\
\hline & $48: 2$ & 18:1_18:1_12:0; 18:1_16:1_14:0; 16:1_16:1_16:0 & $4.1 \pm 1.4$ \\
\hline & $46: 1$ & 12:0_18:1_16:0;14:0_14:0_18:1;16:0_16:1_14:0 & $2.8 \pm 0.9$ \\
\hline \multirow{5}{*}{46} & $56: 5$ & 20:2_16:1_20:2; $18: 1 \_18: 1 \_20: 3$ & $0.3 \pm 0.3$ \\
\hline & $54: 4$ & 18:1_18:1_18:2; 18:2_16:0_20:2 & $3.6 \pm 1.1$ \\
\hline & $52: 3$ & 16:1_18:1_18:1;16:0_18:1_18:2 & $6.5 \pm 0.5$ \\
\hline & $50: 2$ & 18:1_14:0_18:1; 16:0_18:1_16:1 & $8.1 \pm 2.0$ \\
\hline & $48: 1$ & $16: 0 \_16: 1 \_16: 0 ; 14: 0 \_18: 1 \_16: 0$ & $3.5 \pm 1.6$ \\
\hline \multirow{3}{*}{48} & $54: 3$ & 18:1_18:1_18:1 & $7.1 \pm 2.9$ \\
\hline & $52: 2$ & 16:0_18:1_18:1 & $9.7 \pm 0.9$ \\
\hline & $50: 1$ & 16:0_18:1_16:0 & $2.6 \pm 1.2$ \\
\hline \multirow{3}{*}{50} & $56: 3$ & 18:1_18:1_20:1; 18.2_20:0_18:1 & $0.2 \pm 0.2$ \\
\hline & $54: 2$ & 18:1_18:0_18:1; 18:0_18:2_18:0 & $1.3 \pm 0.3$ \\
\hline & $52: 1$ & 16:0_18:1_18:0;18:0_16:1_18:0 & $0.6 \pm 0.3$ \\
\hline \multirow{3}{*}{52} & $58: 3$ & 18:1_18:1_22:1 & $0.1 \pm 0.1$ \\
\hline & $56: 2$ & 18:1_18:1_20:0 & $0.2 \pm 0.1$ \\
\hline & $54: 1$ & 18:0_18:0_18:1;20:0_16:0_18:1 & $0.2 \pm 0.1$ \\
\hline
\end{tabular}

a Shorthand notation of TGs according to Liebisch and co-workers [34]. The most likely FA in the $s n-2$ position of the glycerol is presented as second acid in the notation [28]. 


\section{Experimental}

\subsection{Biological Materials}

Bombus (Megabombus) ruderatus (Fabricius, 1775) males were obtained from laboratory rearing. Colonies were started with females collected in the region of Eastern Prague and settled in the laboratory. When colonies turned to male production, the male imagoes were collected immediately after emergence to avoid possible inbreeding. They were placed in a plastic box together with several workers and supplied with high-quality food. The temperature was kept between $28.5-29.5{ }^{\circ} \mathrm{C}$. For chemical analyses, males were killed by freezing at $-18{ }^{\circ} \mathrm{C}$ during the photophase prior to dissection of the tissues.

The males of Bombus (Ashtonipsithyrus) bohemicus originated from an invaded colony of Bombus lucorum, where the workers were allowed to collect food from nature. Bombus (Metapsithyrus) campestris (Panzer, 1801) males were collected in the Krkonoše Mountains (northeastern Czech Republic, elevation 750-800 m). For chemical analyses, the collected living insects were transported to the laboratory and then stored in a freezer until the fat bodies were dissected. The insect material is deposited in the collection of one of the authors (J.K.).

\subsection{Sample Preparation}

Males of productive age (5-20 days old) were taken from the rearing (B. ruderatus, 14 specimens) or collected in the open air (B. campestris, nine specimens). The labial glands were dissected and extracted with hexane $(200 \mu \mathrm{L}$ per gland). Dissected fat bodies were extracted with chloroform/methanol as previously described [30]. After pre-separation of the lipid extracts using TLC (mobile phase - hexane:diethyl ether:formic acid, 80:20:1), the TG fraction was stored in chloroform solution $(0.1 \mathrm{mg} / 10 \mu \mathrm{L})$ [30] at $-18{ }^{\circ} \mathrm{C}$ prior to analysis. Both labial glands and fat bodies of $B$. ruderatus were dissected, while only the fat bodies of $B$. campestris and B. bohemicus were dissected and analysed in this work. The data obtained from lipid analyses were compared with previously published analytical data for LG secretions from B. campestris and B. bohemicus males [14].

\subsection{GC/MS Analysis of the Labial Gland Extracts}

The extracts were analysed using a gas chromatograph with a splitless injector $\left(220{ }^{\circ} \mathrm{C}\right)$ and mass detector $\left(200{ }^{\circ} \mathrm{C}\right.$, Fisons MD 800 coupled to a Focus GC, Thermo Finnigan S.p.A., Milan, Italy). A DB-5ms column $(30 \mathrm{~m} \times 0.25 \mathrm{~mm}$, film thickness $0.25 \mu \mathrm{m}$, Agilent Technologies $)$ and helium gas (constant flow of $1 \mathrm{~mL} / \mathrm{min}$ ) were used for separations. The temperature program started at $70{ }^{\circ} \mathrm{C}$, after 2 min delay the temperature of the oven was increased to $140{ }^{\circ} \mathrm{C}$ at a rate of $40{ }^{\circ} \mathrm{C} / \mathrm{min}$, then to $240{ }^{\circ} \mathrm{C}$ at a rate of $2{ }^{\circ} \mathrm{C} / \mathrm{min}$, and finally to $320{ }^{\circ} \mathrm{C}\left(15 \mathrm{~min}\right.$ delay) at a rate of $4{ }^{\circ} \mathrm{C} / \mathrm{min}$. Compounds were identified based on their mass spectra as compared to spectra in the National Institute of Standards and Technology Library (NIST, city, state abbrev, USA). Positions of double bonds in unsaturated LG extract components were determined based on preparation of dimethyl disulfide adducts followed by GC/MS analysis [40]. The products were analysed by GC/MS using the same temperature program as for the original extracts. 


\subsection{HPLC/APCI-MS Analysis of TGs}

The HPLC/MS analyses were performed on a system comprising an SMC 1000 vacuum membrane degasser, P 4000 gradient pump, SN 4000 control unit (Thermo Separation Products), and LCQ classic ion-trap mass spectrometer (Thermo Finnigan). Manual injection was accomplished with a Rheodyne type model D injection valve (Ecom, Prague, Czech Republic) equipped with a 5- $\mu \mathrm{L}$ internal injection loop. Two stainless steel Nova-Pak C18 columns $(300 \times 3.9 \mathrm{~mm}, 150 \times 3.9 \mathrm{~mm}$, particle size $4 \mu \mathrm{m}$; Waters) were connected in series and kept at $30{ }^{\circ} \mathrm{C}$. The chromatographic conditions used were developed previously [28] and optimised for bumblebee TG separations [41]. Briefly, non-aqueous reversed phase HPLC gradient separations were performed using acetonitrile (A) and 2-propanol (B) in the mobile phase (gradient: $0 \mathrm{~min}-100 \% \mathrm{~A}$, flow rate of $1 \mathrm{~mL} / \mathrm{min} ; 108 \mathrm{~min}-30 \% \mathrm{~A}, 70 \% \mathrm{~B}, 1 \mathrm{~mL} / \mathrm{min}$; $150 \mathrm{~min}-5 \% \mathrm{~A}, 95 \% \mathrm{~B}, 0.5 \mathrm{~mL} / \mathrm{min} ; 165 \mathrm{~min}-5 \% \mathrm{~A}, 95 \% \mathrm{~B}, 0.5 \mathrm{~mL} / \mathrm{min} ; 177 \mathrm{~min}-100 \% \mathrm{~A}$, $0.5 \mathrm{~mL} / \mathrm{min} ; 180 \mathrm{~min}-100 \% \mathrm{~A}, 1 \mathrm{~mL} / \mathrm{min})$. Ammonium acetate dissolved in 2-propanol/water (1:1) to a final concentration of $100 \mathrm{mM}$ was added post-column at a flow rate of $10 \mu \mathrm{L} / \mathrm{min}$. The APCI source was operated at $400{ }^{\circ} \mathrm{C}$, the heated capillary temperature was $200{ }^{\circ} \mathrm{C}$, and the corona discharge current was set to $4.5 \mu \mathrm{A}$. The full scan mass spectra were recorded in the $m / z$ range of $75-1,300$. Mass spectra were interpreted with the aid of TriglyAPCI software [42]. The peak areas were calculated from reconstructed chromatograms for the $[\mathrm{M}+\mathrm{H}]^{+}$and $\left[\mathrm{M}+\mathrm{NH}_{4}\right]^{+}$ions (both summed together).

\subsection{Transesterification of TG and GC/MS Analysis of FAMEs}

The TG fraction from TLC separation was transesterified using acetylchloride in chloroform $/ \mathrm{methanol}$ as previously described [43]. The reaction mixture was neutralized with silver carbonate. After a brief centrifugation, the supernatant was directly injected into GC/MS, and the resulting FAMEs were identified. Positions of double bonds in unsaturated FAMEs were determined from chemical ionisation mass spectra (reaction gas: acetonitrile) recorded on an ion trap mass analyser (Varian Saturn 2000R, Varian, Walnut Creek, CA, USA) as described in the literature [35].

\section{Conclusions}

Our results show that specific FAs bound in TGs of the male fat body are structurally similar to the major aliphatic components of the male marking pheromone in one bumblebee and two cuckoo-bumblebee species. Thus, our results support the hypothesis that the pheromonal components may be formed from lipidic precursors after their transport to and structural modification in the labial gland. Putting together all results obtained to date, it is likely that both pathways (de novo and transport pathways) are active in the organism, and the dominant one may depend on the physiological state of the animal or the availability of particular precursors.

\section{Supplementary Materials}

Supplementary materials can be accessed at: http://www.mdpi.com/1420-3049/19/2/2330/s1. 


\section{Acknowledgments}

Financial support from the Czech Science Foundation (NO 203/09/1446), the Technology Agency of the Czech Republic (NO TA01020969), and the Institute of Organic Chemistry and Biochemistry (research project RVO: 61388963) is gratefully acknowledged. The authors thank Jaromír Č́ížek for B. ruderatus and B. bohemicus rearing and Oldřich Hovorka for collection of B. campestris specimens. We also thank Pavel Jiroš for his help with transesterifications of the TGs samples and Hillary Hoffman for proof-reading of the manuscript.

\section{Conflicts of Interest}

The authors declare no conflict of interest.

\section{References}

1. Calam, D.H. Species and sex-specific compounds from the heads of male bumblebees (Bombus spp.). Nature 1969, 221, 856-857.

2. Kullenberg, B.; Bergström, G.; Bringer, B.; Calberg, B.; Cederberg, B. Observations on scent marking by Bombus Latr. and Psithyrus Lep. males (Hym., Apidae) and localisation of site of production of the secretion. Zoon Suppl. 1973, 1, 23-25.

3. Bergman, P.; Bergstrom, G. Scent marking, scent origin and species-specificity in male premating behavior of two Scandinavian bumblebees. J. Chem. Ecol. 1997, 23, 1235-1251.

4. Terzo, M.; Urbanová, K.; Valterová, I.; Rasmont, P. Intra and interspecific variability of the cephalic labial glands' secretions in male bumblebees: the case of Bombus (Thoracobombus) ruderarius and B. (Thoracobombus) sylvarum [Hymenoptera, Apidae]. Apidologie 2005, 36, 85-96.

5. Bergman, P. Chemical Communication in Bumblebee Premating Behaviour. Ph.D. Dissertation, Göteborg University, Göteborg, Sweden, 1997.

6. Coppée, A.; Mathy, T.; Cammaerts, M.-C.; Verheggen, F.; Terzo, M.; Iserbyt, S.; Valterová, I.; Rasmont, P. Age-dependent attractivity of males' sexual pheromones in Bombus terrestris (L.) [Hymenoptera, Apidae]. Chemoecology 2011, 21, 75-82.

7. Žáček, P.; Kalinová, B.; Šobotník, J.; Hovorka, O.; Ptáček, V.; Coppée, A.; Verheggen, F.; Valterová, I. Comparison of age-dependent quantitative changes in the male labial gland secretion of Bombus terrestris and Bombus lucorum. J. Chem. Ecol. 2009, 35, 698-705.

8. Kullenberg, B.; Bergström, G.; Ställberg-Stenhagen, S. Volatile components of the cephalic marking secretion of male bumblebees. Acta Chem. Scand. 1970, 24, 1481-1483.

9. Bergström, G.; Svensson, B.G.; Appelgren, M.; Groth, I. Biosystematics of Social Insects; Elsevier: Waltham, MA, USA, 1981; p. 175.

10. Cederberg, B.; Svensson, B.G.; Bergström, G.; Appelgren, M.; Groth, I. Male marking pheromones in North European cuckoo bumble bees, Psithyrus (Hymenoptera, Apidae). Nova Acta Regiae Soc. Ups. 1984, 3, 161-166.

11. Hovorka, O.; Urbanová, K.; Valterová, I. Premating behavior of Bombus confusus males and analysis of their labial gland secretion. J. Chem. Ecol. 1998, 24, 183-193. 
12. Hovorka, O.; Valterová, I.; Rasmont, P.; Terzo, M. Male cephalic labial gland secretions of two bumblebee species of the subgenus Cullumanobombus (Hymenoptera: Apidae: Bombus Latreille) and their distribution in Central Europe. Chem. Biodivers. 2006, 3, 1015-1022.

13. Urbanová, K.; Valterová, I.; Hovorka, O.; Kindl, J. Chemotaxonomical characterisation of males of Bombus lucorum collected in the Czech Republic. Eur. J. Entomol. 2001, 98, 111-115.

14. Urbanová, K.; Halík, J.; Hovorka, O. Kindl, J.; Valterová, I. Marking pheromones of the cuckoo bumblebee males (Hymenoptera, Apoidea, Bombus Latreille): Compositions of labial gland secretions of six species occurring in the Czech Republic. Biochem. Syst. Ecol. 2004, 32, 1025-1045.

15. Terzo, M.; Valterová, I.; Rasmont, P. Atypical secretions of the male cephalic labial glands in bumblebees: The case of Bombus (Rhodobombus) mesomelas Gerstaecker (Hymenoptera, Apidae). Chem. Biodivers. 2007, 4, 1466-1471.

16. Bertsch, A.; Schweer, H.; Titze, A.; Tanaka, H. Male labial gland secretions and mitochondrial DNA markers support species status of Bombus cryptarum and B. magnus (Hymenoptera, Apidae). Insectes Soc. 2005, 52, 45-54.

17. Valterová, I.; Urbanová, K. Chemical signals of bumblebees. Chem. Listy 1997, 91, 846-857.

18. Terzo, M.; Valterová, I.; Urbanová, K.; Rasmont, P. De la nécessité de redécrire les phéromones sexuelles des mâles de bourdons [Hymenoptera, Apidae, Bombini] publiées avant 1996 pour leur utilisation en analyse phylogénétique. Phytoprotection 2003, 84, 39-51.

19. Morgan, D.E. Biosynthesis in Insects; RSC Press: Cambridge, UK, 2004.

20. Tillman, J.A.; Seybold, S.J.; Jurenka, R.A.; Blomquist, G.J. Insect pheromones-An overview of biosynthesis and endocrine regulation. Insect Biochem. Mol. Biol. 1999, 29, 481-514.

21. Matsumoto, S.; Hull, J.J.; Ohnishi, A.; Moto, K.; Fónagy, A. Molecular mechanisms underlying sex pheromone production in the silkmoth, Bombyx mori: Characterization of the molecular components involved in bombykol biosynthesis. J. Insect Physiol. 2007, 53, 752-759.

22. Mann, J. Chemical Aspects of Biosynthesis; Oxford University Press: Oxford, UK, 1994.

23. Lanne, B.S.; Bergström, G.; Wassgren, A.-B.; Törnbäck, B. Biogenetic pattern of straight chain marking compounds in male bumble bees. Comp. Biochem. Physiol. 1987, 88, 631-636.

24. Žáček, P.; Prchalová-Horňáková, D.; Tykva, R.; Kindl, J.; Vogel, H.; Svatoš, A.; Pichová, I.; Valterová, I. De novo biosynthesis of sexual pheromone in the labial gland of bumblebee males. ChemBioChem 2013, 14, 361-371.

25. Luxová, A.; Valterová, I.; Stránský, K.; Hovorka, O.; Svatoš, A. Biosynthetic studies on marking pheromones of bumblebee males. Chemoecology 2003, 13, 81-87.

26. Žáček, P.; Frišonsová, K.; Kindl, J.; Hovorka, O.; Kowalczuk, T.; Valterová, I. Application of the labeled precursors and analysis of their metabolites by means of GCxGC-MS - Tool for biosynthesis of the bumblebee male's sexual pheromone research. ChemPlusChem 2014, submitted.

27. Kofroňová, E.; Cvačka, J.; Vrkoslav, V.; Hanus, R.; Jiroš, P.; Kindl, J.; Hovorka, O.; Valterová, I. Comparison of HPLC/MS and MALDI-MS for characterizing triacylglycerols in insects: Specie-specific composition of lipids in fat bodies of bumblebee males. J. Chromatogr. B 2009, 877, 3878-3884.

28. Jiroš, P.; Cvačka, J.; Hanus, R.; Kindl, J.; Kofroňová, E.; Valterová, I. Changes in composition of triacylglycerols in the fat body of bumblebee males during their lifetime. Lipids 2011, 46, 863-871. 
29. Cvačka, J.; Kofroňová, E.; Vašičková, S.; Stránský, K.; Jiroš, P.; Hovorka, O.; Kindl, J.; Valterová, I. Unusual fatty acids in the fat body of the early bumblebee Bombus pratorum. Lipids 2008, 43, 441-450.

30. Cvačka, J.; Hovorka, O.; Jiroš, P.; Kindl, J.; Stránský, K.; Valterová, I. Analysis of triacylglycerols in fat body of bumblebees by chromatographic methods. J. Chromatogr. A 2006, 1101, 226-237.

31. Appelgren, M.; Bergström, G.; Svensson, B.G.; Cederberg, B. Marking pheromones of Megabombus bumble bee males. Acta Chem. Scand. 1991, 45, 972-974.

32. Rasmont, P.; Terzo, M.; Coppée, A.; Valterová, I. The Species Specific Cephalic Secretions of the Homoplasic and Oligolectic Bumblebees Bombus consobrinus and Bombus gerstaeckeri. In Proceedings of the Third European Conference of Apidology, Belfast, UK, 8-11 September 2008.

33. Valterová, I.; Terzo, M.; Rasmont, P. Institute of Organic Chemistry and Biochemistry, Academy of Sciences of the Czech Republic, Prague, Czech Republic. Unpublished work, 2003.

34. Liebisch, G.; Vizcaíno, J.A.; Köfeler, H.; Trötzmüller, M.; Griffiths, W.J.; Schmitz, G.; Spener, F.; Wakelam, M.J.O. Shorthand notation for lipid structures derived from mass spectrometry. J. Lipid Res. 2013, 54, 1523-1530.

35. Oldham, N.J.; Svatoš, A. Determination of the double bond position in functionalized monoenes by chemical ionization ion-trap mass spectrometry using acetonitrile as a reagent gas. Rapid Commun. Mass Spec. 1999, 13, 331-336.

36. Antony, B.; Fujii, T.; Mot, K.; Matsumoto, S.; Fukuzawa, M.; Nakano, R.; Tatsuki, S.; Ishikawa, Y. Pheromone-gland-specific fatty-acyl reductase in the adzuki bean borer, Ostrinia scapulalis (Lepidoptera: Crambidae). Insect Biochem. Mol. Biol. 2009, 39, 90-95.

37. Tomčala, A.; Bártů, I.; Šimek, P.; Kodrík, D. Locust adipokinetic hormones mobilize diacylglycerols selectively. Comp. Biochem. Physiol. B 2010, 156, 26-32.

38. Bergman, P.; Bergström, G.; Appelgren, M. Labial gland marking secretion in males of two Scandinavian cuckoo bumblebee species (genus Psithyrus). Chemoecology 1996, 7, 140-145.

39. Vioque, J.; Kolattukudy, P.E. Resolution and purification of an aldehyde-generating and an alcohol-generating fatty acyl-CoA reductase from pea leaves (Pisum sativum L.). Arch. Biochem. Biophys. 1997, 340, 64-72.

40. Francis, G.W. Alkylthiolation for the determination of double bond positions in unsaturated fatty acid esters. Chem. Phys. Lipids 1981, 29, 369-374.

41. Kofroňová, E.; Cvačka, J.; Jiroš, P.; Sýkora, D.; Valterová, I. Analysis of insect triacylglycerols using liquid chromatography-Atmospheric pressure chemical ionization mass spectrometry. Eur. J. Lipid Sci. Technol. 2009, 111, 519-525.

42. Cvačka, J.; Krafková, E.; Jiroš, P.; Valterová, I. Computer-assisted interpretation of atmospheric pressure chemical ionization mass spectra of triacylglycerols. Rapid Commun. Mass Spectrom. 2006, 20, 3586-3594.

43. Stránský, K.; Jursík, T. Simple quantitative transesterification of lipids. 1. Introduction. Fett/Lipid 1996, 98, 65-71.

Sample Availability: Not available.

(C) 2014 by the authors; licensee MDPI, Basel, Switzerland. This article is an open access article distributed under the terms and conditions of the Creative Commons Attribution license (http://creativecommons.org/licenses/by/3.0/). 